\title{
Meeting reports
}

\section{IN vitro toxicology: From INtestine to bralN}

\author{
doi:10.14573/altex.1706151
}

On February 23, 2017, a second meeting was held at the University of Agricultural Sciences and Veterinary Medicine (USAMV) in Cluj-Napoca, Romania, with representatives of the Romanian Center for Alternative Test Methods (ROCAM), the Italian Platform on Alternative Methods (IPAM), and the Italian Association of in vitro Toxicology (CELLTOX). The meeting aimed at promoting the $3 \mathrm{Rs}$ in education (Daneshian et al., 2011; Sachana et al., 2014) by giving an overview on the use of in vitro models for testing substances through a multidisciplinary vision and approach.

Adela Pintea, University of Agricultural Sciences and Veterinary Medicine, Cluj-Napoca, presented a lecture on the activities of the Cell Culture Laboratory of the Institute of Life Sciences UASMV, ROCAM member, where the main objective is the use of in vitro tests for the evaluation of the biological activity of natural compounds, including polyphenols, carotenoids, and antioxidant compounds. Retinal pigmented epithelial (RPE) cells were used under conditions that mimic oxidative stress in the human retina, such as generation of hydrogen peroxide during phagocytosis, hyperglycemia, and in the presence of photosensitizers. Lutein, zeaxanthin, resveratrol and some phenolic acids were found to protect RPE D407 cells either by direct inactivation of reactive oxygen species or by enhancing the level of reduced glutathione and the activity of antioxidant enzymes. A novel strategy to increase the bioavailability of natural compounds is the development of new formulations, such as multilayer polyelectrolyte microcapsules (PEM). Microcapsules loaded with phenolic compounds (resveratrol, anthocyanins) were successfully produced using biocompatible materials. Resveratrol containing PEM were not cytotoxic through a wide range of concentrations and were internalized by RPE cells, as demonstrated by confocal microscopy, transmission electron microscopy (TEM), and scanning electron microscopy (SEM). Fractions from anthocyanin-rich sources (blueberries, chokeberries, elderberries), purified and characterized by powerful analytical techniques, were tested in various cell culture models using normal or tumor cell lines. Besides the well-known antioxidant properties, anthocyanins from chokeberries showed antidiabetic properties by stimulation of insulin secretion in $\beta$-TC3 murine pancreatic cells. Antiproliferative and proapoptotic properties of anthocyanins were also observed in B16-F10 murine melanoma cells and in HeLa human tumor cervical cells. A recent interest is the use of an in vitro cell model for the evaluation of cellular uptake and cytotoxicity of gold nanoparticles with possible applications as surface enhanced Raman spectroscopy (SERS)-active tags or substrates inside living cells.

Francesca Caloni, University of Milan (DIMEVET), emphasized the importance of the use of species-specific in vitro models in veterinary toxicology. Predictive models and tools in human, environmental and veterinary toxicology are urgently required in order to satisfy the $3 \mathrm{Rs}-$ Reduce, Refine, Replace - for risk assessment evaluation. In vitro tests are necessary not only as stand-alone methods but, even more, in Integrated Testing Strategy (ITS) approaches for the understanding of complex pathways of toxicity in order to assess the adverse effects of substances. 3D cultures and tissues, co-cultures, organoids and organ-on-a-chip approaches, which are increasingly complex models used for toxicological risk assessment thorough Integrated Approaches to Testing and Assessment (IATA), have been developed. Species-specific in vitro barriers, mimicking oral, respiratory and topical exposure are predictive for absorption, bioavailability, transport and toxicity of compounds, both in human and veterinary pharmaceutical and toxicological studies. Models used in veterinary studies include dog skin, porcine alveolar and intestinal epithelial cells and primary bovine mammary epithelial cells (pbMEC) cultured on semi-permeable inserts; measured endpoints include cytotoxicity, barrier integrity and marker release. Recently, an interesting 3D feline hepatic organoid was developed (Kruitwagen et al., 2017).

Teresa Coccini (ICS Maugeri - IRCCS Pavia), in videoconference, explained that her institute has acquired large experience in toxicological research conducted using an in vitro testing strategy based on the use of complementary cell-based assays for the assessment of the health impact of several xenobiotics related to occupational and environmental exposure and consumer safety. The selected methods provide fast responses to a range of doses (lethal \& sub-lethal) and times of exposure (acute \& prolonged) in human cell lines representative of the in vivo target organ (i.e., CNS) regarding a wide range of endpoints relevant in terms of mechanisms of action known to be triggered by potential neurotoxicants (such as mitochondrial function, membrane integrity, cell death pathways, cell cycle progression, oxidative stress, etc.). The in vitro testing models for neurotoxicity studies include (i) 2D mono-cultures of human astrocytes (astrocytoma D384 cells) and neurons (neuroblastoma SH-SY5Y cells). Several results have been obtained using this approach, aimed at elucidating the mechanistic pathways involved in the neurotoxicity caused by new and emerging materials such as iron oxide $\left(\mathrm{Fe}_{3} \mathrm{O}_{4} \mathrm{NPs}\right)$ nanoparticles (Coccini et al., 
2017); (ii) 2D co-cultures of human neurons (SH-SY5Y cells) and astrocytes (D384 cells) using a Transwell system where cell types are separated by a semi-permeable membrane. This model recapitulates the tissue microenvironment and mimics the physiological conditions in the brain where neurons are surrounded by glial cells. Ongoing studies are devoted to setting-up 3D cell cultures for each cell type (i.e., D384 and SH-SY5Y) mimicking tissue architecture and cell communications using ultra-low attachment 96-well round bottomed plates. The obtained spheroids, which display spontaneous self-organization, can recreate aspects of the spatial and mechanical environment of the CNS that closely resemble in vivo conditions and thus provide useful tools for neuroscience studies. In parallel, the group is pursuing the use of mesenchymal stem cells derived from human umbilical cord blood, which have the potential to allow production of a virtually limitless supply of normal human cells that can be developed into many different cell types, including astrocytes and neurons.

Flaviu Tabaran, University of Agricultural Sciences and Veterinary Medicine, Cluj-Napoca, gave a presentation entitled "Carbon and gold nanoparticles: insight into cellular and tissue detection methods and short-term toxicity". The unique physicochemical properties of nanomaterials promise fascinating opportunities for research in medical sciences. A critical aspect in experiments using nanomaterials is represented by the difficulty of identifying them in biological samples. The presentation discussed the analytical methods and the algorithms employed in the detection of carbon nanotubes (CNT) and gold nanoparticles (GNP) in cells and tissues. The advantages and limitations of TEM and atomic force microscopy, micro-Raman spectroscopy, classical optical and high-resolution dark-field microscopy were described with respect to their relevance in in vitro and systemic toxicity studies. A strong correlation was found between the distribution of such materials and their local adverse effect.

A discussion with the audience on methodologies and perspectives both regarding the application and the development of the models concluded the meeting, confirming the importance of the collaboration between ROCAM, IPAM, CELLTOX and the host institution.

We thank Cristina Cortinovis for her contribution to the organization of the meeting.

\section{References}

Caloni, F., Meloni, M., Sambuy, Y. et al. (2016). New alternative models for in vitro toxicology. ALTEX 33, 470-471. doi:10.14573/altex.1607251

Coccini, T., Caloni, F., Ramirez Cando, L. J. et al. (2017). Cytotoxicity and proliferative capacity impairment induced on human brain cell cultures after short- and long-term exposure to magnetite nanoparticles. J Appl Toxicol 37, 361-373. doi:10.1002/jat.3367

Daneshian, M., Akbarsha, M. A., Blaauboer, B. et al. (2011). A framework program for the teaching of alternative methods (replacement, reduction, refinement) to animal experimentation. ALTEX 28, 341-352. doi:10.14573/altex.2011.4.341

Kruitwagen, H. S., Oosterhoff, L. A., Vernooij, I. G. W. H. et al. (2017). Long-term adult feline liver organoid cultures for disease modeling of hepatic steatosis. Stem Cell Reports 8, 822-830. doi:10.1016/j.stemcr.2017.02.015

Sachana, M., Theodoridis, A., Cortinovis, C. et al. (2014). Student perspectives on the use of alternative methods for teaching in veterinary faculties. Altern Lab Anim 42, 223-233.

\section{Claudia Diana Gherman ${ }^{1}$, Cornel Catoi ${ }^{2}$, Carmen Socaciu ${ }^{2}$,} Adela Pintea ${ }^{2}$, Nechita Adrian Oros ${ }^{2}$, Flaviu Tabaran ${ }^{2}$, Andras-Laszlo Nagy ${ }^{2}$, Yula Sambuy ${ }^{3}$, Isabella De Angelis ${ }^{4}$, Teresa Coccini ${ }^{5}$, Virginia Bassi ${ }^{6}$ and Francesca Caloni ${ }^{6}$

1University of Medicine and Pharmacy "Iuliu Hatieganu", Cluj-Napoca, Romania; ${ }^{2}$ University of Agricultural Sciences and Veterinary Medicine, Romanian Center for Alternative Test Methods (ROCAM), Cluj-Napoca, Romania; ${ }^{3}$ CREA, Food \& Nutrition Research Center, Rome, Italy; ${ }^{4}$ Istituto Superiore di Sanità, ISS, Department of Environment and Primary Prevention, Rome, Italy; ${ }^{5}$ Laboratory of Clinical and Experimental Toxicology, Toxicology Unit, Maugeri Clinical Scientific Institutes SpA-BS, IRCCS Pavia; 'Università degli Studi di Milano, Department of Veterinary Medicine (DIMEVET), Milan, Italy 